\title{
Communications
}

\section{Facile Preparation of Trimethylsulfonium Bromide}

\author{
Koon Ha Park, ${ }^{*}$ Myung Sub So, ${ }^{\dagger}$ and Yong Whan Kim* \\ Department of Chemistry, Chungnam National University, Daejeon 305-764, Korea. *E-mail: khpark@cnu.ac.kr \\ ${ }^{\dagger}$ Kunsan Jeil High School, Kunsan, Chonbuk 573-410, Korea \\ Received August 1, 2005
}

Key Words : Kornblum oxidation, Trimethylsulfonium bromide, Dimethyl sulfoxide, Benzyl bromide

Trimethylsulfonium bromide, utilized to make oxiranes ${ }^{1}$ and molten salts, ${ }^{2}$ has been reported to be prepared from dimethyl sulfoxide and ethyl bromoacetate, ${ }^{3,4}$ alkyl halide and dimethyl sulfide, ${ }^{5}$ and dimethyl sulfoxide and bromine. ${ }^{1}$ Herein, we report a very simple and convenient method of trimethylsulfonium bromide from a modified Kornblum oxidation condition. ${ }^{6}$

Typical Kornblum oxidation is referred to a reaction in which alkyl/aryl halide (tosylate) is reacted with dimethyl sulfoxide followed by a direct workup procedure in basic aqueous solution to prepare corresponding aldehyde (path 1 in Figure 1). ${ }^{7-11}$ However we have found that Kornblum oxidation in the absence of water and base afforded colorless crystalline trimethylsulfonium bromide in about $65 \%$ yields after recrystallization. The X-ray crystallographic and NMR data of trimethylsulfonium bromide were consistent with those of the published report. ${ }^{3}$

Several pathways can be envisaged for the formation of trimethylsulfonium bromide in our conditions. However we believe that a path 2 in Figure 1 could be a major route in our acidic conditions based on control experiments. We found out that volatile products (methyl bromide, dimethyl sulfide, and hydrogen bromide) were efficiently made in our conditions. That is, we were able to isolate a mixture of aniline hydrobromide and N-methylaniline which was formed in an aniline trap attached at the outlet of reflux condenser. In an another experiment we could observe and collect solid trimethylsulfonium bromide (about $1 \mathrm{gm}$ ) formed in a reservoir attached between the reaction flask and reflux condenser, which gave us an information that methyl bromide and dimethyl sulfide were obviously generating in an appreciable amount in our reaction conditions.

Supposing a path 2 in Figure 1 to be a major route, then one might ask for the verification of benzyl methanesulfenate. We tried to get any evidence for the existence of benzyl methanesulfenate by both NMR and GC/MS, only to fail. We believe that benzyl methanesulfenate can not survive our acidic conditions. Instead we found out that benzyl methyl sulfide and dibenzyl sulfide were formed.

In order to get more pieces of evidence related with this modified Kornblum oxidation reactions, reactions of benzyl bromide with diphenyl sulfoxide and methyl phenyl sulf-

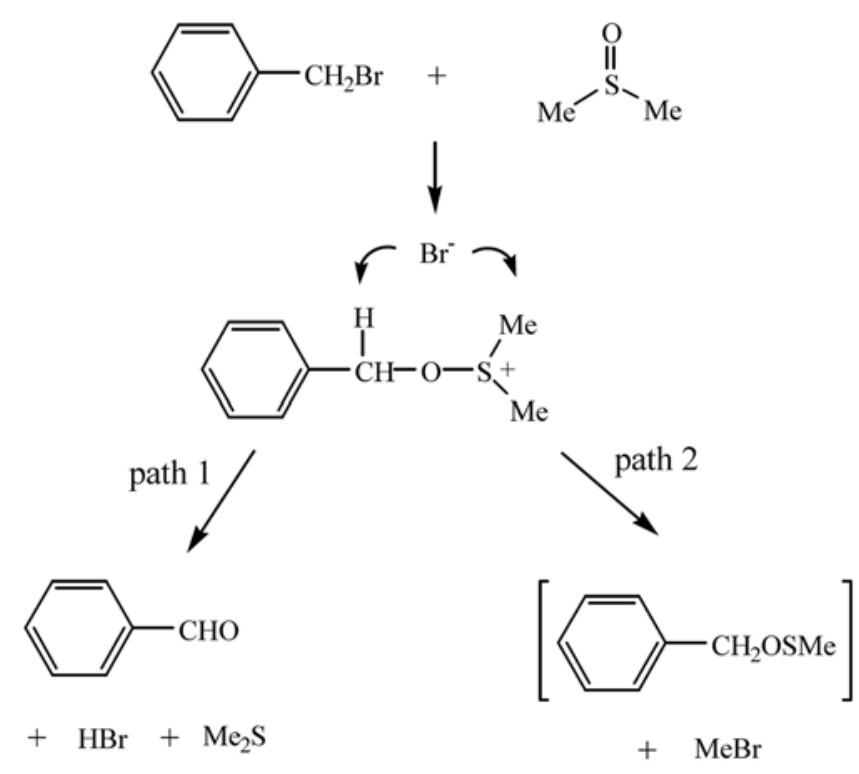

Figure 1. Reaction of benzyl bromide and dimethyl sulfoxide.

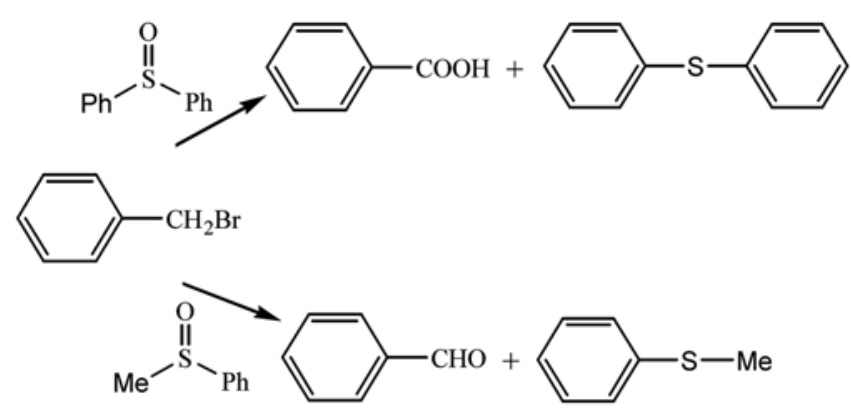

Figure 2. Reactions of benzyl bromide and other sulfoxides.

oxide were carried out.

As shown in Figure 2, reactions of benzyl bromide with either diphenyl sulfoxide and methyl phenyl sulfoxide turned out to be very clean by NMR. Trimethylsulfonium bromide was not formed at all in these reactions. Noteworthy was that further oxidation proceeded in reaction of diphenyl sulfoxide to give benzoic acid, instead of benzaldehyde obtained in the reaction of methyl phenyl 
sulfoxide.

In overall an interesting feature in the reaction of benzyl bromide with dimethyl sulfoxide without a base and water is the high yields of trimethylsulfonium bromide we obtained $(65 \%)$. We believe that hydrogen bromide generated by path 1 in Figure 1 would contribute to make more dimethyl sulfide in reaction with dimethyl sulfoxide as reported in the previous literature. ${ }^{12}$ Then this extra dimethyl sulfide can be used to make trimethylsulfonium bromide.

Acknowledgement. This work was supported by nondirective research program of Chungnam National University (2003).

\section{References}

1. (a) Munavu, R. M.; Ogur, J. A. Kenya J. Sci. Technol. Ser. A 1980, 1(2), 111. (b) Corey, E. J.; Chaykovsky, M. J. Am. Chem. Soc. $\mathbf{1 9 6 5}, 87,1353$

2. (a) Xiao, L.; Johnson, K. E. Can. J. Chem. 2004, 82(4), 491. (b) Welton, T. Chemical Rev. 1999, 99, 2071.
3. Svensson, Per H.; Kloo, L. Acta Cryst. C 1996, C52(10), 2580.

4. Major, R. T.; Hess, H. J. J. Org. Chem. 1958, 23, 1563.

5. Truce, W. E.; Badiger, V. V. J. Org. Chem. 1964, 29, 3277.

6. Typical procedure is as follows. A solution of benzyl bromide $(2.38 \mathrm{~mL}, 20 \mathrm{mmol})$ and dimethyl sulfoxide $(10 \mathrm{~mL})$ contained in a round bottomed flask equipped with condenser was heated at 80 ${ }^{\circ} \mathrm{C}$ for 3 hours. When the reaction mixture was cooled to room temperature, trimethylsulfonium bromide was precipitated which was washed with acetone thoroughly. Recrystallization from acetonitrile afforded colorless crystals. Yield $2.05 \mathrm{~g}(65 \%) \mathrm{mp}$ 198-200 ${ }^{\circ} \mathrm{C}(\mathrm{dec}) .{ }^{1} \mathrm{H}$ NMR (DMSO- $\left.d_{6}\right) \delta 2.9(s)$.

7. Kornblum, N.; Jones, W. J.; Anderson, G. J. J. Am. Chem. Soc. 1959, 81, 4113.

8. Kornblum, N.; Powers, J. W.; Anderson, G. J.; Jones, W. J.; Larson, H. O.; Levand, O.; Weaver, W. M. J. Am. Chem. Soc. 1957, 79, 6552 .

9. Nace, H. R.; Monagle, J. J. J. Org. Chem. 1959, 24, 1792.

10. Helms, A.; Heitler, D.; McLenden, G. J. Am. Chem. Soc. 1992 , $114,6227$.

11. Godfrey, A. G.; Ganem, B. Tetrahedron Lett. 1974, 11, 917.

12. Majetich, G.; Hicks, R.; Reister, S. J. Org. Chem. 1997, 62, 4321 and reference 5 therein. 